\title{
Spectrum and Dynamics of the BCS-BEC Crossover from a Few-Body Perspective.
}

\author{
Javier von Stecher and Chris H. Greene \\ Department of Physics and JILA, University of Colorado, Boulder, Colorado 80309-0440
}

\begin{abstract}
The spectrum of two spin-up and two spin-down fermions in a trap is calculated using a correlated gaussian basis throughout the range of the BCS-BEC crossover. These accurate calculations provide a few-body solution to the crossover problem. This solution is used to study the time-evolution of the system as the scattering length is changed, mimicking experiments with Fermi gases near FanoFeshbach resonances. The structure of avoiding crossings in the spectrum allow us to understand the dynamics of the system as a sequence of Landau-Zener transitions. Finally, we propose a ramping scheme to study atom-molecule coherence.
\end{abstract}

PACS numbers:

Optical lattices are a powerful tool to study few body systems. When tunneling is negligible, optical lattices can be viewed as an ensemble of individual harmonic traps where the properties of these systems can be studied. The interaction between the particles can be tuned using a Fano-Feshbach resonance [1] and the number of particles in each lattice site can be controlled [2, 3]. In a recent experiment with optical lattices, the spectrum of two fermions in a trap has been measured [2], demonstrating that few body trapped systems can be studied in their own right. Also, the BCS-BEC crossover has been routinely explored in experiments with ultracold Fermi

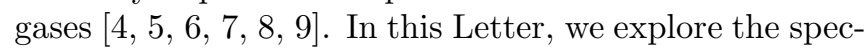
trum and dynamics of four trapped particles and we show how a few-body formulation allows us to obtain accurate solutions of the system without making the standard approximations of many-body theory. This provides an explicit representation of avoided crossings between the atomic degenerate Fermi gas (DFG or BCS)-type states and molecular BEC-type states. Our results directly apply to optical lattice experiments and they provide a fewbody perspective on BCS-BEC crossover dynamics.

Specifically, we calculate the spectrum of two pairs of trapped fermionic atoms interacting through short-range potentials, all with the same mass $m$. One pair is assumed to be distinguishable from the other pair, but the two atoms within each pair are indistinguishable. The s-wave scattering length $a$ of the short-range interactions will be tuned in the standard manner, [1] which allows us to explore the BCS-BEC crossover as a function of interaction strength near a broad Fano-Feshbach resonance. Even though the BCS theory is not expected to apply to a 4-particle system, we still use this term to refer to the dynamical regime where $a$ is small and negative. By solving the problem from a few-body perspective, we are able to give accurate properties - especially energy levels as well as time-dependent dynamics - of the full quantum mechanical spectrum at zero temperature. As a result we achieve a deeper understanding of the global topology of the spectrum, in addition to making quantitative predictions of transition probabilities and dynamical properties of this system when interactions change with time as in experiments [4, [5, [6, 7, [8, ].

To obtain the energy spectrum, we use a correlated gaussian basis set [10, 11]. A diabatization procedure reduces the system to a tractable number of relevant eigenfunctions, after which we solve the time-dependent Schrödinger equation using the diabatic representation.

The Hamiltonian adopted is

$$
\mathcal{H}=\sum_{i}^{4}\left(-\frac{\hbar^{2}}{2 m} \nabla_{i}^{2}+\frac{1}{2} m \omega_{0}^{2} \mathbf{r}_{i}^{2}\right)+\sum_{i=1}^{2} \sum_{j=3}^{4} V\left(\mathbf{r}_{i j}\right) .
$$

In this convention, particles 1 and 2 are "spin up" and particles 3 and 4 are "spin down". The two-body potential function $V\left(\mathbf{r}_{i j}\right)$ is taken to be a purely attractive gaussian, $V\left(\mathbf{r}_{i j}\right)=V_{0} \exp \left(-\mathbf{r}_{i j}^{2} / 2 d_{0}^{2}\right)$ where the width $d_{0}$ of the gaussian is fixed and the depth $V_{0}$ is tuned to produce the desired two-body scattering length $a$. To obtain results independent of the model potential properties, we concentrate on the range $d_{0} \ll a_{h o}$, where $a_{h o}=\left(\hbar / m \omega_{0}\right)^{1 / 2}$ is the trap length. By considering different widths $d_{0}$ ranging from $0.05 a_{h o}$ to $0.01 a_{h o}$ we have verified that our results exhibit a weak dependence on $d_{0}$. All results presented in this Letter correspond to $d_{0}=0.01 a_{h o}$. The eigenspectrum of Eq. (11) is obtained by an expansion into correlated Gaussian basis set, i.e.,

$$
\Psi_{\left\{d_{i j}\right\}}\left(\mathbf{r}_{1}, \mathbf{r}_{2}, \mathbf{r}_{3}, \mathbf{r}_{4}\right)=\mathcal{S}\left\{\psi_{0}\left(\mathbf{R}_{C M}\right) e^{-\sum_{j>i} r_{i j}^{2} / 2 d_{i j}^{2}}\right\}
$$

with $\mathbf{R}_{C M}=\left(\mathbf{r}_{1}+\mathbf{r}_{2}+\mathbf{r}_{3}+\mathbf{r}_{4}\right) / 4$ the center-ofmass coordinate, $\psi_{0}$ the center-of-mass ground state $\psi_{0}\left(\mathbf{R}_{C M}\right)=e^{-2 R_{C M}^{2} / a_{h o}^{2}}$, and $\mathcal{S}$ the symmetrization operator. Opposite-spin fermions are treated as distinguishable particles, so $\mathcal{S}=\left(1-\mathcal{P}_{1,2}\right)\left(1-\mathcal{P}_{3,4}\right)$ where $\mathcal{P}$ is the permutation operator. The wavefunctions obtained from this basis set are in the ground center-of-mass state (with $J_{C M}=0$ ). The relative coordinate wavefunction has quantum numbers $J^{\pi}=0^{+}$, since the basis functions only depend on $\mathbf{R}_{C M}$ and the interparticle distances, $r_{i j}$.

The basis functions, defined in Eq.(2), are characterized by the set of values $\left\{d_{i j}\right\}$ which are selected semirandomly. While the $d_{i j}$ corresponding to different spin fermions are selected to range from a fraction of $d_{0}$ up to 
a couple of times $a_{h o}$ to describe dimer formation, those $d_{i j}$ corresponding to equal spin fermions are selected to be of the order of the trap length $a_{h o}$. The typical size of the basis set used in these calculations is about 700015000. The advantage of the correlated gaussian basis set is that all the matrix elements can be calculated analytically in terms of the $\left\{d_{i j}\right\}$ 's and the properties of the two-body potential and the trap. Prior to diagonalizing the Hamiltonian, a linear transformation eliminates linear dependence, typically reducing the basis set size by less than $10 \%$. The basis set is fixed while $V_{0}$ is tuned to give different scattering lengths, whereby matrix elements are calculated once and then used to obtain the spectrum throughout the entire range of the BCS-BEC crossover.

The accuracy and convergence of the calculations have been verified in detail. The ground state energy agrees with fixed-node diffusion Monte Carlo (FN-DMC) calculations throughout the BCS-BEC crossover [12]. For example, the ground state energy at unitarity varies from $5.099 \hbar \omega$ to $5.027 \hbar \omega$ as we change $d_{0}$ from $0.05 a_{h o}$ to $0.01 a_{h o}$; FN-DMC calculations for a square well potential of range $0.01 a_{h o}$ lead to a ground state energy $5.069(9) \hbar \omega$ [12]. The difference between our results and the FNDMC results is thus about $1 \%$, where the shape and range of the interactions start to play a role. Higher excited states are in agreement with the BCS and BEC limiting behaviors. Furthermore, the ground and excited states in the BEC can be used to extract the dimer-dimer scattering length, which agrees with the Petrov et al. prediction [13], and the corresponding effective range [12].

The spectrum as a function of $\lambda \equiv 1 / a$ shows a series of apparent crossings and avoided crossings in the unitarity region. The avoided crossings can be roughly characterized by their width $\Delta \lambda$, the range over which the two adiabatic eigenstates interact appreciably, into two main categories: narrow crossings, where $\Delta \lambda \ll 1 / a_{h o}$, and wide crossings, where $\Delta \lambda \gtrsim 1 / a_{h o}$. We adopt a variant of the diabatization procedure presented in Ref. [14 to diabatize narrow crossings while leaving wide crossings adiabatic, which gives smooth energy curves. Figure 1 presents the partially-diabatic spectrum in the BCS-BEC crossover. The inset shows a zoom of the transition region, i.e., the strongly interacting regime where the avoiding crossings occur. This structure of avoiding crossings permits a global view of the manner in which states evolve from weakly interacting fermions at $a<0$ to all the different configurations of a Fermi gas at $a>0$, i.e. molecular bosonic states, fermionic states and molecular boson-Fermi mixture (see Fig. 11). Furthermore, it allow us to visualize concretely the possible pathways of the time-dependent sweep experiments, as is shown below.

A partially-diabatic representation can be used to describe a ramp of an initial configuration through the BCS-BEC crossover as in the experiments carried out at different laboratories, like JILA and Rice. The ini-

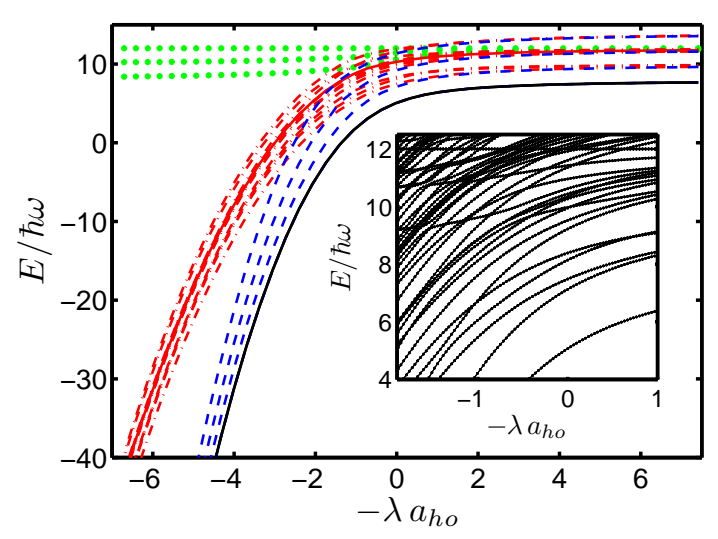

FIG. 1: (Color Online) The energy spectrum for four particles in a trap is shown versus the dimensionless quantity $\lambda a_{h o}(20$ states that correlate diabatically with the 20 lowest energies in the noninteracting limit). The black solid curve is the ground state. The blue dashed curves are states that diabatically approach excited dimer-dimer configurations, the red dashdotted curves correspond to states that represent one dimer plus two free atoms on the BEC side, while the states in green circles correlate diabatically to four free atoms. The lowest curve drawn with green circles is the Fermi gas ground state on the BEC side of the resonance. Inset: zoom of the adiabatic spectrum in the crossover regions. In this figure, all states are considered, showing the rich structure of avoiding crossings.

tial configuration is propagated using the time dependent Schrödinger equation. Starting from the ground state in the BCS side, the parameter $\lambda$ is ramped through the resonance to the BEC side at different speeds $\nu=\frac{d \lambda}{d t}$. In a homogenous system, the ramps are only characterized by the initial density $\rho$, the speed $\nu$ and $m$. This suggests that $\chi \equiv \frac{m}{\hbar \rho}\left|\frac{d \lambda}{d t}\right|$ is the relevant dimensionless quantity that characterize the ramp speed in large systems. Thus, we will use $\chi$ to compare 4 -body results with large systems. This idea of using the density, and not properties of the trap, to connect few-body calculations with large systems has been previously implemented [15].

To interpret our numerical results we apply the Landau-Zener approximation, which predicts that the probability for a transition from the adiabatic $\Psi_{j}$ to $\Psi_{i}$ is $T_{i j}(\chi)=e^{-\varkappa_{i j} / \chi}$, where $\varkappa_{i j}$ are dimensionless parameters extracted from properties of the adiabatic eigenstates, as is discussed below.

The nonadiabatic coupling or $P$-matrix controls the probability of nonadiabatic transitions to a good approximation using the Landau-Zener model. The coupling between two adiabatic states $\Psi_{i}$ and $\Psi_{j}$ is $P_{i j}(\lambda) \equiv$ $\left\langle\Psi_{i} \mid \frac{\partial \Psi_{j}}{\partial \lambda}\right\rangle$ where $\lambda$ is the adiabatic parameter. Clark has shown that, if the transition has a form consistent with the Landau-Zener approximation, then the $P$ matrix element for a transition from $\Psi_{j}$ to $\Psi_{i}$ has a Lorentzian form whose width, along with the correspond- 
ing eigenenergies, characterize the Landau-Zener parameter $\varkappa_{i j}$ [16]. We numerically evaluate all the potentially important $P_{i j}$ and verify that the largest couplings generally have a smooth single-peak form that is approximately Lorentzian. The largest $P_{i j}$ relevant for this specific dynamical sweep correspond to transitions among $\Psi_{1}, \Psi_{2}, \Psi_{5}$ and $\Psi_{14}$. Here, the partially-diabatic states are labeled in increasing order of their energy on the BCS side (see Fig. 10. $\Psi_{1}$ refers to the ground state, $\Psi_{2}$ is the first excited dimer-dimer configuration, $\Psi_{5}$ is the second configuration with a dimer and two atoms, and $\Psi_{14}$ is the lowest configuration with no dimers, the "fermionic ground state on the BEC side". The final probability distribution can be explained as sequence of Landau-Zener transitions between these four partially-diabatic states where the positions of the peaks of the $P_{i j}(\lambda)$ determine a specific order in which the transitions occur, namely $1 \rightarrow 2$, followed by $2 \rightarrow 5$ and $1 \rightarrow 5$, and finally $5 \rightarrow 14$.

We use $p_{i}$ to denote the probability of ending up in state $\Psi_{i}$ following one ramp that started out in the DFG ground state (\#1) on the BCS side. This Landau-Zener model then predicts that

$$
\begin{gathered}
p_{1}=\left(1-T_{5,1}\right)\left(1-T_{2,1}\right), \\
p_{2}=\left(1-T_{5,2}\right) T_{2,1}, \\
p_{5}=\left(1-T_{14,5}\right)\left[T_{5,1}\left(1-T_{2,1}\right)+T_{5,2} T_{2,1}\right], \\
p_{14}=T_{14,5}\left[T_{5,1}\left(1-T_{2,1}\right)+T_{5,2} T_{2,1}\right] .
\end{gathered}
$$

The sum of all these probabilities is unity by construction. The Landau-Zener parameters obtained from the P-matrix analysis are $\varkappa_{2,1} \approx 35, \varkappa_{5,1} \approx 43, \varkappa_{5,2} \approx 14$ and $\varkappa_{14,5} \approx 90$. Interestingly, the Landau-Zener model shows decent agreement with the numerical results despite the neglect of many possible transitions. Figure 2 presents the probability of evolving in a certain configuration following a single unidirectional ramp, as a function of the dimensionless ramp speed parameter $\chi$. The numerical ramps are initiated at $\lambda_{i} \sim-7 / a_{h o}$ and finalized at $\lambda_{f} \sim 7 / a_{h o}$. As the speed is increased, the final state changes from a molecular bosonic ground state, i.e., $\Psi_{1}$, to a "fermionic ground state", i.e., $\Psi_{14}$, in qualitative agreement with experiments.

To relate our results with experiments carried out at JILA and Rice, we write the Landau-Zener parameter for atom-molecule transitions $\delta=\varkappa_{m o l} / \chi$ in terms of experimentally accessible variables. If the dependence of $a$ on the magnetic field is approximated in the usual manner as $a(B)=a_{b g}\left(1+\frac{w}{B-B_{0}}\right)$ then $\nu=(d B / d t) / w a_{b g}$ (measured near unitarity, i.e., where the transitions occur). Therefore, $\left.\delta=\varkappa_{m o l}(d B / d t)^{-1} \rho \hbar \mid w a_{b g}\right\rfloor / m$ which agrees with previous theoretical predictions [17, 18]. The dependence of $\delta$ on $\rho$ has been experimentally verified [19]. To evaluate $\chi$ we use the average density for the noninteracting 4-particles Fermi gas, namely $\rho \approx 0.153 / a_{h o}^{3}$. The molecular fraction, the fraction of atoms that become molecules after the ramp is over, is probably the

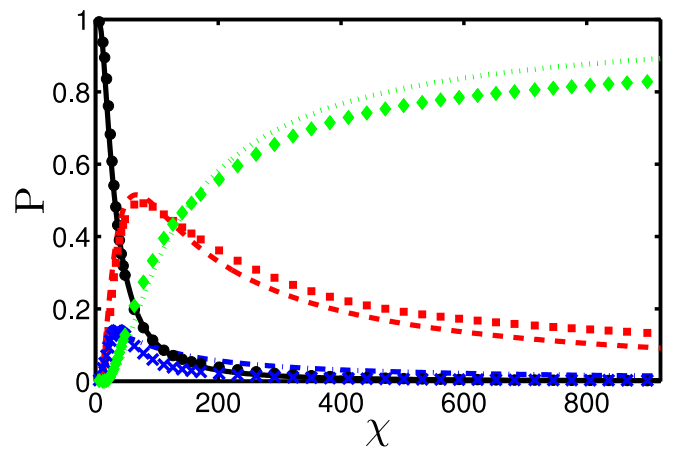

FIG. 2: (Color Online) The probability of evolving into a given configuration is shown as a function of the dimensionless ramp speed parameter $\chi$. The symbols correspond to the full numerical solution while the curves are Landau-Zener results. The black solid curve and circles correspond to the ground state configuration. The blue dash-dotted curve and crosses correspond to higher dimer-dimer excitations. The red dashed curve and squares correspond to ramps that produce a dimer plus two free atoms. The green dotted curve and diamonds correspond to the lowest configuration of four free atoms, i.e., the Fermi gas "ground state" on the BEC side of the resonance.

most relevant quantity to compare with experiments. For our 4-body system, the molecular fraction is defined as the probability of ending up in a dimer-dimer configuration plus half of the probability to form a configuration of the "dimer plus two free atom" type. In the experiments we compare with, the molecule fraction was fitted to a Landau-Zener function, i.e. $p_{m o l}^{L Z}=f_{m}\left(1-e^{-\varkappa_{m o l} / \chi}\right)$, where $f_{m}$ is is the maximum conversion efficiency which depends on temperature.

Whether a Landau-Zener function is the correct functional form to describe the molecule formation fraction in large systems remains a question that existing experiments have not resolved [18, 20, 21]. The Landau-Zener model presented in this work for four particles does not predict a single Landau-Zener function but a combination of different Landau-Zener terms. However, the final molecule fraction predicted by this model and the numerical results for the molecule formation fraction can be approximately fitted by this Landau-Zener function with $\varkappa_{m o l}^{(4)} \approx 59 \pm 6$; this value is higher than the twobody prediction of $\varkappa_{\text {mol }}^{(2)} \approx 42$. Our results are consistent with the experimental Landau-Zener parameter obtained in Ref. [4] for ${ }^{40} \mathrm{~K}$. The fit of the experimental data to a Landau-Zener formula predicted a $\varkappa_{\text {mol }}^{\text {exp }} \approx 62 \pm 15$. Also, experiments carried out at Rice measured the LandauZener parameter for ${ }^{6} \mathrm{Li}$ [7]. Taking into account the conditions of the experiment and the properties of the ${ }^{6} \mathrm{Li}$ Fano-Feshbach resonance at $B \approx 543.8 G$, we estimate $\varkappa_{\text {mol }}^{e x p} \sim 90$. Both experiments were carried out at finite temperature and, consequently, maximum conversion efficiencies were approximately $f_{m} \sim 0.5$, while our calculations are at $T=0$ where $f_{m}=1$. In summary, 
both experiments are in general agreement with the fourbody predictions. In the following, we propose more sophisticated sweeps to study atom-molecule coherence.
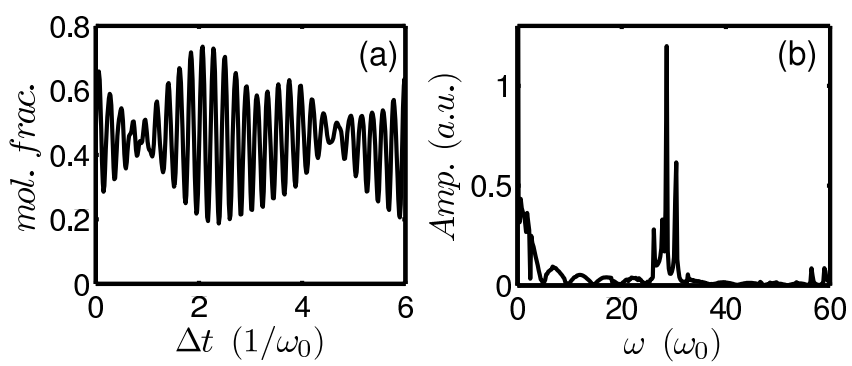

FIG. 3: (a) Molecular formation fraction is shown as a function of the delay $\Delta t$. (b) Fourier transform of the Fig.3(a). The peaks of the spectrum correspond to the most important energy transitions.

Atom-molecule oscillations 22] or quantum beats 15] have previously been explored for condensates, and also for fermionic systems near a narrow Fano-Feshbach resonance 23]. To study atom-molecule coherence in a Fermi gas near a broad Fano-Feshbach resonance, we have considered different ramping schemes and noticed one that enhances the atom-molecule oscillations. Starting in the ground state on the BCS side, one ramps at medium speed $(\chi \sim 80)$ to the BEC side and pauses for a time $\Delta t$ at a value $\lambda_{\text {still }}$; then one ramps back at the same speed to the BCS side where the scattering length is close to zero. Finally, one slowly ramps $\lambda$ to the $\operatorname{BEC}(\chi \sim 7)$ side and measures the resulting molecular fraction. This is shown as a function of $\Delta t$ in Fig. 3(a). Observe that this ramping scheme produces large coherent oscillations in the molecular fraction. To interpret their frequencies we Fourier transform the time-dependent molecular fraction (see Fig. 3(b)). The frequency domain peaks correlate with the most important configurations during the waiting period at $\lambda_{\text {still }} \sim 5 / a_{h o}$. The Bohr frequencies at $\omega \approx 28 \omega_{0}$ correspond to coherences between states differing in one broken dimer bond. For example, the highest peak is a coherence between $\Psi_{5}$ and $\Psi_{14}$ while the second highest is a coherence between $\Psi_{1}$ and $\Psi_{5}$. The frequencies around $\omega \approx 57 \omega_{0}$ correspond to coherences between states differing in two broken dimers bonds, e.g. coherences between $\Psi_{1}$ and $\Psi_{14}$, and between $\Psi_{2}$ and $\Psi_{14}$. In optical lattice experiments, this kind of multipeak structure should be particularly pronounced in a tight trap.

The four body problem remains fundamental and challenging. We have presented an accurate numerical solution of the spectrum of two "spin up" and two "spin down" fermions in a trap throughout the range of the BCS-BEC crossover. Even though the spectrum presents a rich structure of avoided crossings, we have shown that a simple Landau-Zener model approximately describes the dynamics of unidirectional ramps. The spectrum and dynamics of this system is interesting for optical lattice experiments. These would allow access to physics that cannot be probed in the two-body system, like corrections to the energy spectrum due to the dimerdimer interaction, and also to the atom-dimer interaction. Also, the system of two "spin up" and two "spin down" fermions in a trap exhibits many of the ingredients of the BCS-BEC crossover problem, and in that sense the present results provide a few-body perspective on Fermi gas experiments.

We are grateful to Doerte Blume for providing unpublished data to us, and Josh Dunn, Seth Rittenhouse, Nirav Mehta and Jose D'Incao for useful discussions. This work was supported in part by NSF.

[1] S. Inouye et al., Nature (London) 392, 151 (1998). S. Cornish et al., Phys. Rev. Lett. 85, 1795 (2000). M. Greiner, C. Regal, and D. Jin, Nature 426, 537 (2003).

[2] T. Stoferle et al., Phys. Rev. Lett. 96, 030401 (2006).

[3] Y. Miroshnychenko et al., Phys. Rev. Lett. 97, 243003 (2006).

[4] C. A. Regal et al., Nature 424, 47 (2003).

[5] S. Jochim et al., Science 302, 2101 (2003).

[6] M. W. Zwierlein et al., Phys. Rev. Lett. 91, 250401 (2003).

[7] K. E. Strecker, G. B. Partridge, and R. G. Hulet, Phys. Rev. Lett. 91, 080406 (2003).

[8] T. Bourdel et al., Phys. Rev. Lett. 93, 050401 (2004).

[9] J. Kinast et al., Phys. Rev. Lett. 92, 150402 (2004).

[10] K. Singer, Proc. R. Soc. London, Ser. A 258, 412 (1960).

[11] K. Varga and Y. Suzuki, Comp. Phys. Com. 106, 157 (1997).

[12] J. von Stecher, C. H. Greene, and D. Blume, condmat/0705.0671.

[13] D.S. Petrov, C. Salomon, G. V. Shlyapnikov, Phys. Rev. Lett. 93, 090404 (2004).

[14] M. Hesse, A. T. Le, and C. D. Lin, Phys. Rev. A 69, 052712 (2004).

[15] B. Borca, D. Blume, and C.H. Greene, New J. of Phys. 5, 111 (2003).

[16] C. Clark, Phys. Lett. 70A, 295 (1979).

[17] K. Goral et al., J. Phys. B 37, 3457 (2004).

[18] J. Williams, N. Nygaard, and C. Clark, New J. of Phys. 8, 150 (2006).

[19] E. Hodby et al., Phys. Rev. Lett. 94 , 120402 (2005).

[20] E. Pazy et al., Phys. Rev. Lett. 95, 170403 (2005).

[21] E. Altman and A. Vishwanath, Phys. Rev. Lett. 95, 110404 (2005).

[22] E. A. Donley et al., Nature 417, 529 (2002).

[23] A. Andreev, V. Gurarie, and L. Radzihovsky, Phys. Rev. Lett. 93, 130402 (2004). 